\title{
6
}

\section{FROM DUST TO COMETS}

\author{
J. M. GREENBERG
}

The growth and chemical evolution of a typical interstellar dust grain are followed starting from average interstellar conditions to the dense cloud and contraction phase. Based on the theory of cometary accretion directly from cold interstellar dust it is shown that the bulk material of a primordial comet would consist mostly of an icy conglomerate of complex organic molecules and frozen radicals in which are imbedded approximately equal volumes (10\% each) of small grains in two different sizes - one variety being the order of $0.05 \mu$ and the other $0.005 \mu$ in size - consisting mostly of silicates

\section{INTRODUCTION}

The major portion of the discussion in this meeting has been devoted to consideration of condensation processes in the pre-solar nebula starting from atoms and working up through molecules to solids. As we know, these atoms must have originated in an interstellar cloud of gas and dust and one of the key questions to be answered is how much of the heavy element material in the various bodies of the solar system is fully recycled from the primordial dust via evaporation and recondensation and how much may be a direct relic.

In this paper we shall assume some of the bodies in the solar system such as comets - may have condensed directly out of the primordial dust. It is therefore necessary first to describe in some detail the dust properties as they may have existed in the pre-pre solar nebula. On this basis one arrives at a comet model which contains the same chemical elements as the classical "icy" comet but in substantially modified combinations. See Whipple (1976) for a brief history of comet theories with references.

\section{AVERAGE INTERSTELLAR DUST AND GAS}

In order to describe the dust and gas in the latest stage of interstellar cloud contraction we shall follow the development of a typical interstellar grain and its associated gas from some arbitrary point of reference, namely an average as defined observationally.

Al though many theoretical dust models have successfully matched the amount and the wavelength dependence of extinction of star light, there is only one which has simultaneously produced the correct wavelength dependence of interstellar extinction and linear and circular polarization in the wavelength range up to $\lambda^{-1} \approx 3 \mathrm{\mu m}^{-1}$. Within this region, hereafter referred to as the classical part, the extinction and linear and circular polarization may be produced by elongated core-mantle particles with silicate cores of thickness about $0.1 \mu \mathrm{m}$ 
and mantles with a diameter about $0.25 \mathrm{\mu m}$, consisting of some sort of ices . Detailed calculations have been performed for a number of models (Greenberg and Hong 1974a, Hong 1975) but for our purposes here the differences are of no significance. In any case the best representation consists of concentric cylindrical grains (the cylinder representation is dictated by computational availability) with silicate cores of $0.05 \mu \mathrm{m}$ radius and icelike mantles distributed in size according to

$$
n\left(a_{m}\right)=\exp \left[-5\left(\frac{a_{m}-a_{c}}{a_{i}}\right)^{3}\right]
$$

with $a_{i}=0.20 \mu \mathrm{m}$.

It may be shown that the mantle size distribution with cutoff $a_{i}$ is equivalent, for our purposes, to a single size mantle $\bar{a}_{m} \approx 0.3 a_{i}=0.12 \mu \mathrm{m}$.

In addition to the classical sized grains there must be a large number of very small grains in order to provide the far ultraviolet extinction. These grains, of somewhat uncertain chemical composition - possibly silicates or graphite - are observationally determined to be of the order of $0.003-0.005 \mu \mathrm{m}$ radius and as a consequence of their smallness may be shown on both physical and observational grounds to provide a hostile basis for mantle accretion and therefore remain bare.

There are two mechanisms that inhibit mantle growth on the very small particles. One of them is temperature fluctuations (Greenberg and Hong 1974b) induced by absorption of ultraviolet photons and the other is temperature fluctuations induced by molecule formation on the surface. Should the very small particles as well as the classical core-mantle particles add mantles of only $0.002 \mathrm{~m}$ thickness the combined extinction curve in the visual region would look like that produced by smaller particles rather than that produced by larger particles as is actually observed (Carrasco et al. 1973). The discussion on the evolution of dust grains in dark clouds will therefore be almost completely limited to following the classical sized grains.

It is important to note that the classical sized grains generally have very low temperatures, of the order of $T_{d}=10 \mathrm{~K}-15 \mathrm{~K}$ and significantly lower in the centers of dark clouds.

\section{THE DEPLETION OF HEAVY ELEMENTS IN THE INTERSTELLAR MEDIUM}

A general feature of the interstellar medium is the high correlation between gas and dust. Since the dust is made up almost exclusively of elements heavier than hydrogen, but excluding helium, we expect that the gas will be depleted in these relative to hydrogen. Table I shows for a particular star how much of the more common heavy atomic species $\mathrm{C}, \mathrm{N}, \mathrm{O}$, Si, $\mathrm{Mg}$, Fe have heen observed in the gas (Morton 1975) and how much is in the solid grains as compared with the amount expected to be found hased on cosmic abundance (Cameron 1973). As was shown earlier (Greenherg 1974) with a slightly different grain model, the general tendency is that a significant number of the $0 . C$. N group is not ac. counted for. Furthermore any grain model which excludes the ices imposes an even greater discrepancy hetween the observed abundances and the cosmic abundances of the $O, C, N$ group. Finally, it may he demonstrated that effects of grain shape do not make any significant changes in our calculation of dust bound atoms (Greenberg and Hong 1975) and that if any modifications must be made they are in the interpretation based on curves of growth produced by interstellar absorption of some of the atomic species in the gas. It must be emphasized that this depletion mystery is necessary if we are to believe that grains hecome larger in dense clouds because without the left over (whether accounted for or not) condensible atoms there is not enough material in the gas to change the grain sizes as much as is ohserved. 


\section{FROM DUST TO COMETS}

TABLE I

DUST AND GAS ATOMIC DEPLETION

\begin{tabular}{|c|c|c|c|c|c|c|}
\hline \multirow[b]{3}{*}{ Core } & \multirow{2}{*}{$\begin{array}{l}\text { r. Oph } \\
\mathrm{Mg} / \mathrm{H}\end{array}$} & \multicolumn{5}{|c|}{$\begin{array}{c}\mathrm{N}_{\mathrm{h}} / \mathrm{A}(\mathrm{V})=2.34 \times 10^{21} \text { atoms } \mathrm{cm}^{-2} \mathrm{mag}^{-1} \\
\text { Atom to Hydrogen Ratio }\left(10^{-5}\right)\end{array}$} \\
\hline & & $\mathrm{Fe} / \mathrm{H}$ & $\mathrm{Si} / \mathrm{H}$ & $\mathrm{O} / \mathrm{H}$ & $\mathrm{C} / \mathrm{H}$ & $\mathrm{N} / \mathrm{H}$ \\
\hline & 1.6 & 1.3 & 1.5 & 5.8 & --- & $\cdots$ \\
\hline Mantle & --- & --- & -- - & 12.9 & 7.1 & 2.2 \\
\hline Bare & 2.2 & 1.9 & 2.0 & 8.1 & --- & $-\cdots$ \\
\hline Total & 3.8 & 3.2 & 3.5 & 26.8 & 7.1 & 2.2 \\
\hline $\begin{array}{l}\text { Cosmic } \\
\text { Abundance }\end{array}$ & 3.34 & 2.80 & 3.14 & 67.6 & 37.1 & 11.7 \\
\hline
\end{tabular}

$\begin{array}{lrrrrrr}\text { Dust } & 1.14 & 1.14 & 1.11 & 0.40 & 0.19 & 0.19 \\ \text { Gas } & 0.04 & 0.05 & 0.03 & 0.30 & 0.21 & 0.23 \\ \text { Total } & 1.18 & 1.19 & 1.14 & 0.70 & 0.40 & 0.42 \\ \text { Missing } & -0.18 & -0.19 & -0.14 & 0.30 & 0.60 & 0.58\end{array}$

\section{CHEMICAL MODIFICATION OF INTERSTELLAR GRAINS BY ULTRAVIOLET PHOTOPROCESSING}

\section{A. Interstellar Grain Photolysis}

The low temperature icy mantles of the classical grains provide an excellent environment for the production and storing of free radicals created hy ultraviolet photolysis. Free radicals like co could also he directly accreted out of the gas. The first question to be answered is whether the rate of free radical production is such that the time required to produce a significant number in an individual grain is substantially smaller than the characteristic life cycle of $-10^{7}-10^{8}$ years for an interstellar cloud of dust and gas.

If we estimate the net efficiency of a photon with energy greater than $5 \mathrm{eV}$ producing a free radical in a typical grain as being only $10^{-3}$ we arrive at a time of the order of only $10^{3}$ years to produce a $1 \%$ density (Greenberg 1976).

Given a $1 \%$ free radical density, there is then a high probability that a chain reaction between these highly reactive species may be induced in the mantle material. Should two free radicals approach each other, they immediately combine because the reaction almost certainly requires zero activation energy The energy released by the reaction goes in part to heating the grain material which thus releases neighbouring radicals from their sites to approach still other radicals, etc. Since the grain lifetime is so much longer than the time for free radical production we may expect that this photoprocessing may orcur thousands of times in the life of each grain. Consequently, no matter what the chemical composition of the mantle is at any one time, the average grain would be found to contain a kind of steady state mixture of large and small molecules as well as free radical combinations of the atoms of cxygen, carbon and nitrogen with hydrogen.

The mechanism of photolysis of grains by interstellar ult raviolet radiation certainly leads to the creation of organic molecules much larger than any which have yet been observed in interstellar space (Snyder 1976) and the limiting molecule size may he as large as the mantle itself. If the energy released in a chain reaction occurs when the dust grain is already at an elevated temperature, as in regions of new star formation, the entire grain may explode (or 
evaporate) leading to the ejection of many very large complex molecules into space some of which degrade hy photodissociation to the sizcs more readily ohserved.

B. Laboratory Studies of Interstellar Grain Photolysis

The study of chemical kinetics in a low temperature solid of the complexity of the icy grain mantle is at best in its infancy. The problems are very complex and, although theoretical calculations of a very crude sort may he used to show the relevance to interstellar grains, there is a need for many new lahoratory investigations. In the fall of 1970 a series of experiments involving the ultraviolet radiation of cosmic abundance mixtures of water. methane and ammonia deposited on the cold finger of a cryostat at $30 \mathrm{~K}$ were hegun by the author and his collaborators (Greenberg et al. 1972). This work has continued at the State University of New York at Albany and now an additional and completely new laboratory is being built in Laboratory Astrophysics at the University of Leiden. Recent work on irradiation of mixtures of water, ethane and ammonia at $77 \mathrm{~K}$ with the $1640 \AA$ line of bromine have yielded significant products of butane, ethyl alcohol, and ethyl amine. Irradiation of methylamine at $77 \mathrm{~K}$ with the $2062 \&$ line of iodine has yielded dimethylamine $(M=45)$, as well as very abundant amounts of molecules with molecular weights 51 and 88 (not yet identified). These are merely two examples of the fact that break up and recombination of the molecules in ices resulting from photodissociation is readily achieved. Further work will be done at much lower temperatures $(-10 \mathrm{~K})$, using shorter wavelengths. In addition to making gas chromatograph-mass spectrometer identification of the resulting products after evaporation, infrared spectra of the irradiated materials will be used as a diagnostic of the processes taking place in situ within the solid. We also have some expectation that identifications may eventually be made with infrared spectra taken of the interstellar medium. The emphasis is on the processes taking place within the solid rather than on the surface although the latter effects are certainly not to be ignored.

A propos of comets the study of x-ray irradiation of low temperature ices has been looked at very preliminarily (Greenberg et al. 1972) and will be pursued further. In this case the effects are much more rapid (in the laboratory) and quite dramatic. Our one experiment conducted several years ago using $x$-rays on the water, ethane, ammonia mixture led to the production of copious quantities of many sizes of molecules, the most abundant being Co (expected) and next a molecule (unidentified) with molecular weight $M \sim 350$ ! It is important to note that the resultant of the irradiated sample was largely nonvolatile at room temperature and had to be dissolved off the cold finger before it could be studied in the mass spectrometer. This implies that the interstellar grain mantle material is likely to be much more refractory than has been assumed for ices made of water and other simple molecules.

\section{DENSE CLOUDS}

Let us now follow an average dust grain in a cloud of ever increasing density. It is generally accepted that at dust temperatures of $\mathrm{T}_{\mathrm{d}}=10 \mathrm{~K}$ any atom or molecule which strikes the grain will stick with almost unit probability. The temperature of the gas in an $\mathrm{HI}$ cloud is found to range from about $\mathrm{T}_{\mathrm{g}}=$ $100 \mathrm{~K}$ for clouds with $\mathrm{n}_{\mathrm{H}+\mathrm{H}_{2}}=10 \mathrm{~cm}^{-3}$ to $\mathrm{T}_{\mathrm{g}}=20-40 \mathrm{~K}$ in clouds with $\mathrm{n}_{\mathrm{H}+\mathrm{H}_{2}} z$ $10^{3} \mathrm{~cm}^{-3}$. By $\mathrm{n}_{\mathrm{H}+\mathrm{H}_{2}}$ we mean the number of hydrogen atoms in both atomic and molecular form. Assuming a sticking factor of unity for all condensible atoms and starting with a mean grain radius of $0.12 \mu \mathrm{m}$ how large and how fast can a grain grow? The former question is answered by taking the ratio of the volume of the missing plus detected $\mathrm{O}, \mathrm{C}$, and $\mathrm{N}$ atoms plus the neon atoms (neon con- 
denses on dust in very dense clouds) to the volume of the mantle material of the average grain. This ratio is about 6.5 leading to (as calculated for spherical grains) a mantle size ratio of 1.8 and therefore a maximum mean mantle radius of about $0.22 \mu \mathrm{m}$. A preliminary estimate of the variation of the maximum wavelength dependence of polarization on mean size indicates a relationship $\lambda \mathrm{p} \max ^{-1} \overline{\mathrm{a}}_{\mathrm{m}} \approx 0.24$. Presuming that the accretion of grains in dark clouds occurs at a rate faster than cloud collapse (see next section) we may anticipate that grains in regions of new star formation will initially have mantle thicknesses as great or greater than twice those of normal grains. Thus the fact that the wavelength dependence of both extinction and polarization in the Trapezium region (Breger 1974) indicates substantially larger than normal grains is not too surprising since the additional thickness is substantially less than the maximum possible and allows for some mantle losses following the heating due to star formation. However, the values of $\lambda_{\mathrm{p}} \max \approx 8000 \AA$ for $\rho$ Oph given in Carrasco et al. (1973) imply a mean mantle radius of $\bar{a}_{m} \approx 0.20$ which is well on the way to approaching the limiting value, having depleted $\approx 3 / 4$ of all the condensible atoms, including neon.

\section{CLOUD CONTRACTION VERSUS GRAIN ACCRETION}

If, in the process of cloud contraction leading ultimate to star formation, the time for contraction is shorter than that for grain accretion, there would be no possibility of the grains approaching their maximum size. We shall compare these two time scales to determine whether this requirement for total or almost. total condensible atom depletion is reasonable.

The free fall time used to represent the rate of cloud contraction is given by:

$$
{ }_{\mathrm{ff}}=\left(\frac{3}{4 \pi G_{\rho}}\right)^{\frac{1}{2}}=\frac{4 \times 10^{7}}{n_{H^{\frac{1}{2}}}} \mathrm{yrs} .
$$

where we have used $\rho=n_{H} m_{H}+n_{H e} m_{H e}=1.4 n_{H} m_{H}$

The accretion time is given by some factor (of the order of unity) times the mean collision time for a condensible atom to strike any grain in the cloud. The area of the grains per unit area along an atom path is given by the extinction in the visual divided by the optical efficiency factor. Since the latter has been precisely calculated for the core-mantle cylinder model (Hong 1975) to be 1.13 we may let it be unity in our rather crude calculation. Then the accretion time is given by

$$
\tau_{a c}=\frac{A^{\frac{1}{2}} N_{H} / A(V)}{v_{H}}
$$

where $A=$ an average atomic weight of the condensible species, and $v_{H}=$ the velocity of a hydrogen atom. In the early stages the gas temperature is about $100 \mathrm{~K}$ and decreases, as the cloud contracts, to perhaps as low as $20 \mathrm{~K}$ leading to a decrease in $v_{H}$ by about $1 / 2$. In the meanwhile, the total grain surface area grows (by ultimately as much as a factor of almost 4 ) so that the product $v_{H} A(V)$ is a slowly increasing function which varies by less than a factor of 2 during the cloud contraction grain accretion process.

Using the initial condition of $\mathrm{T}=100 \mathrm{~K}, \mathrm{~A} \approx 25, \mathrm{~N}_{\mathrm{H}} / \mathrm{A}(\mathrm{V})=2.34 \times 10^{21}$ atoms $\mathrm{cm}^{-2} \mathrm{mag}^{-1}$ we get

$$
\tau_{\text {ac }}=\frac{2.6 \times 10^{9}}{n_{H+H_{2}}} \text { yrs. }
$$




$$
\frac{{ }^{\tau} \mathrm{ac}}{{ }_{\mathrm{\tau} f \mathrm{ff}}}=0.65 \times 10^{2} \mathrm{n}_{\mathrm{H}+\mathrm{H}_{2}}^{-\frac{1}{2}}
$$

This means that when a cloud reaches a density of $\mathrm{n}_{\mathrm{H}+\mathrm{H}_{2}}=10^{4} \mathrm{~cm}^{-3}$ the accretion rate is faster than the collapse rate. This should have important consequences in the dynamics of cloud contraction and will certainly affect the ionization of a dark cloud as well as its temperature.

The first stage of cloud contraction and the question of grain-grain sticking are well known problems for which a number of solutions have been offered and to which we contribute only a consideration of the state of the dust and gas pre-existing this phase. Entering this phase we would predict that a cloud contains two kinds of grains. Those which are the sub-micron core-mantle type with much thicker than average mantles $\left(a_{m} \approx 0.22 \mu\right.$ ) and those bare particles of the order of $0.005 \mu$ in size which are presumed to be silicates and/or graphite. The mantles, having been subjected to previous ultraviolet radiation contain a substantial density of stored radicals. In the process of heating accompanying star formation some of the grains may shed all or portions of their mantles in the form of complex organic molecules. Those grain mantles which are not fully evaporated will consist mostly of complex molecules which are certainly more refractory than the classical ices. Finally those grains which remain far enough from the source of heating will continue to carry the stored radicals.

If comets accrete directly from the dust there are two possible alternative chemical compositions depending on the heating history of the grains.

1) An agglomeration of silicate core-icy mantle grains in which the mantles contain a large amount of stored energy in the form of frozen radicals. Intermixed with these particles are the bare particles which contribute approximately as much to the total volume as the cores. However the mantles would contribute more than $80 \%$ of the cometary material.

2) Same as above except that the density of stored radicals is low. In this case the cometary ices are a mixture of complex molecules.

During the course of this meeting, Frofessor Whipple pointed out to me that regardless of this choice of original chemical composition, a substantial thickness of the comets surface has undergone further high energy photon processing this time by interstellar x-rays - during its billion or so year lifetime and should therefore consist of a mixture of complex molecules as well as stored free radicals.

The existence of stored radicals to some depth in the cometary ices is a possible source of the energy required to produce those occasional cometary outbursts which occur as the comet warms up in its approach towards the sun. We see from the above that there are several historical paths leading to the likelihood of this composition and it is therefore important to perform a variety of experiments both in the laboratory and in space as well as further observations in order to establish the validity of this model.

\section{ACKNOWLEDGEMENTS}

The recent laboratory results on ultraviolet radiation of low temperature ices have been communicated by Dr. A. J. Yencha. I should like also to thank Dr. S. S. Hong for helpful discussions. 


\section{REFERENCES}

Breger, M. 1974, Planets, Stars and Nebulae Studied with Photopolarimetry, (Ed. T. Gehrels), Univ. of Arizona Press, 946.

Cameron, A.G.W. 1973, Space Science Rev., 15, 121.

Carrasco, L., Strom, S. E., and Strom, K. M. 1973, Ap.J., 182, 95.

Greenberg, J. M. 1974, Ap.J., 189, L81.

Greenberg, J. M. 1976, Astrophysics and sp. Sci., 39, 9.

Greenberg, J. M., and Hong, S. S. 1974a, Galactic Radio Astronomy, Proc. I.A.U.

Symp. 60, Ed. F. J. Kerr, and S. C. Simonson, III (Reide1, Dordrecht) 155.

Greenberg, J. M., and Hong, S. S. 1974b, HII Regions and the Galactic Center,

Proc. of 8th ESLAB Symposium, Ed. A.F.M. Moorwod, ESRO SP-105, 153.

Greenberg, J. M., and Hong, S. S. 1975, The Dusty Universe, ed. G. B. Field, and A.G.W. Cameron, N. Watson Academic Publications, 132.

Greenberg, J. M., Yencha, A. J., Corbet, J. W., and Frisch, H. L. 1972, Memoires de la Societé Royale des Sciences de Liège, be sèrie, tome III, 425.

Hong, S. S. 1975, Ph.D. Thesis, State University of New York at Albany.

Morton, D. C. 1975, Ap.J., 197, L85.

Snyder, L. 1976, private communication.

Whipple, F. L. 1976, Nature, 263, 15.

\section{DISCUSSION}

WILLIAMS: You quoted $0.2 \mu \mathrm{m}$ as the largest observed grain; does this mean that you disbelieve Rowan-Robinson's observations of 100 um grains in the orion Nebula?

GREENBERG: Not necessarily. What happens to these maximum size but still submicron grains later in the star formation process is still uncertain. If they can further combine with each other to make comets $I$ do not see why they might not also make the large "snow" balls of Rowan-Robinson.

HARTMANN: Your talk viewed interstellar grains as precursors of planetary material. We can be fairly sure that pulverized silicate and icy grains are blown out of forming planetary systems (or even present solar system). (See my review in Scientific American, Sept., 1975). Therefore, can we turn the problem around and view planetary systems as precursors of grains? Is it a cyclic process: Grains concentrate in clouds - clouds collapse to form stars and condense (planetary) matter - stars blow out planetary matter to form grains?

GREENBERG: There is no requirement for additional core material ( 0.05 $\mu$ silicate particles) ejected from star formation processes if we believe inferences from $M$ - super-giant mass losses which appears to provide all the core material we need. However, this does not preclude the possibility that some of the interstellar grains after being totally evaporated - not just mantle evaporation - provide the heavy elements some of which are condensed in the form of small solid particles and ejected from the solar system. Since most of the material in a collapsing cloud is not recycled in star formation processes, I would expect that the dust we see around young regions of star formation is mostly residual dust and not reconstituted dust. 